\title{
Detection of variable VHE $\gamma$-ray emission from the extra-galactic $\gamma$-ray binary LMC P3
}

H.E.S.S. Collaboration, H. Abdalla ${ }^{1}$, A. Abramowski ${ }^{2}$, F. Aharonian ${ }^{3,4,5}$, F. Ait Benkhali ${ }^{3}$, E. O. Angüner ${ }^{21}$, M. Arakawa ${ }^{42}$, C. Armand $^{24}$, M. Arrieta $^{15}$, M. Backes ${ }^{8}$, A. Balzer ${ }^{9}$, M. Barnard ${ }^{1}$, Y. Becherini ${ }^{10}$, J. Becker Tjus ${ }^{11}$, D. Berge ${ }^{37}$, S. Bernhard ${ }^{13}$, K. Bernlöhr ${ }^{3}$, R. Blackwell ${ }^{14}$, M. Böttcher ${ }^{1}$, C. Boisson ${ }^{15}$ J. Bolmont ${ }^{16}$, S. Bonnefoy ${ }^{37}$, P. Bordas ${ }^{3}$, J. Bregeon ${ }^{17}$, F. Brun ${ }^{26}$, P. Brun ${ }^{18}$, M. Bryan ${ }^{9}$, M. Büchele ${ }^{36}$, T. Bulik ${ }^{19}$, M. Capasso ${ }^{29}$, S. Caroff ${ }^{30}$, A. Carosi $^{24}$, S. Casanova ${ }^{21,3}$, M. Cerruti ${ }^{16}$, N. Chakraborty ${ }^{3}$, R. C. G. Chaves ${ }^{17,22}$, A. Chen ${ }^{23}$, J. Chevalier ${ }^{24}$, S. Colafrancesco ${ }^{23}$, B. Condon ${ }^{26}$, J. Conrad ${ }^{27,28}$, I. D. Davids ${ }^{8}$, J. Decock ${ }^{18}$, C. Deil ${ }^{3}$, J. Devin ${ }^{17}$, P. deWilt ${ }^{14}$, L. Dirson ${ }^{2}$, A. Djannati-Atai ${ }^{31}$, A. Donath ${ }^{3}$, L.O'C. Drury ${ }^{4}$, J. Dyks ${ }^{34}$, T. Edwards ${ }^{3}$, K. Egberts ${ }^{35}$, G. Emery ${ }^{16}$, J.-P. Ernenwein ${ }^{20}$, S. Eschbach ${ }^{36}$, C. Farnier ${ }^{27,10}$, S. Fegan ${ }^{30}$, M. V. Fernandes ${ }^{2}$, A. Fiasson ${ }^{24}$, G. Fontaine ${ }^{30}$, S. Funk ${ }^{36}$, M. Füßling ${ }^{37}$, S. Gabici ${ }^{31}$, Y. A. Gallant ${ }^{17}$, T. Garrigoux ${ }^{1}$, F. Gaté ${ }^{24}$, G. Giavitto ${ }^{37}$, D. Glawion ${ }^{25}$, J. F. Glicenstein ${ }^{18}$, D. Gottschall ${ }^{29}$, M.-H. Grondin ${ }^{26}$, J. Hahn ${ }^{3}$, M. Haupt ${ }^{37}$, , J. Hawkes $^{14}$, G. Heinzelmannn ${ }^{2}$,

G. Henri ${ }^{32}$, G. Hermann ${ }^{3}$, J. A. Hinton ${ }^{3}$, W. Hofmann ${ }^{3}$, C. Hoischen ${ }^{35}$, T. L. Holch ${ }^{7}$, M. Holler ${ }^{13}$, D. Horns ${ }^{2}$, A. Ivascenko ${ }^{1}$, H. Iwasaki ${ }^{42}$, A. Jacholkowska ${ }^{16}$, M. Jamrozy ${ }^{38}$, D. Jankowsky ${ }^{36}$, F. Jankowsky ${ }^{25}$, M. Jingo ${ }^{23}$, L. Jouvin ${ }^{31}$, I. Jung-Richardt ${ }^{36}$, M. A. Kastendieck ${ }^{2}$, K. Katarzyński ${ }^{39}$, M. Katsuragawa ${ }^{43}$, U. Katz ${ }^{36}$, D. Kerszberg ${ }^{16}$, D. Khangulyan ${ }^{42}$, B. Khélifi ${ }^{31}$, J. King ${ }^{3}$, S. Klepser ${ }^{37}$, D. Klochkov ${ }^{29}$, W. Kluźniak ${ }^{34}$, Nu. Komin ${ }^{23, \star}$, K. Kosack ${ }^{18}$, S. Krakau ${ }^{11}$, M. Kraus ${ }^{36}$, P. P. Krüger ${ }^{1}$, H. Laffon ${ }^{26}$, G. Lamanna ${ }^{24}$, J. Lau ${ }^{14}$, J. Lefaucheur ${ }^{15}$, A. Lemière ${ }^{31}$, M. Lemoine-Goumard ${ }^{26}$, J.-P. Lenain ${ }^{16}$, E. Leser $^{35}$, T. Lohse ${ }^{7}$, M. Lorentz $^{18}$, R. Liu ${ }^{3}$, R. López-Coto ${ }^{3}$, I. Lypova ${ }^{37}$, D. Malyshever9, V. Marandon ${ }^{3}$, A. Marcowith ${ }^{17}$, C. Mariaud ${ }^{30}$, R. Marx ${ }^{3}$, G. Maurin ${ }^{24}$, N. Maxted ${ }^{14,44}$, M. Mayer ${ }^{7}$, P. J. Meintjes ${ }^{40}$, M. Meyer ${ }^{27,46}$, A. M. W. Mitchell ${ }^{3}$, R. Moderski ${ }^{34}$, M. Mohamed ${ }^{25}$, L. Mohrmann ${ }^{36}$, K. Morä $^{27}$, E. Moulin ${ }^{18}$, T. Murach ${ }^{37}$, S. Nakashima ${ }^{43}$, M. de Naurois ${ }^{30}$, H. Ndiyavala ${ }^{1}$, F. Niederwanger ${ }^{13}$, J. Niemiec ${ }^{21}$, L. Oakes ${ }^{7}$, P. O'Brien ${ }^{33}$, H. Odaka ${ }^{43}$, S. Ohm $^{37}$, M. Ostrowski ${ }^{38}$, I. Oya ${ }^{37}$, M. Padovani ${ }^{17}$,

M. Panter ${ }^{3}$, R. D. Parsons ${ }^{3}$, N. W. Pekeur ${ }^{1}$, G. Pelletier ${ }^{32}$, C. Perennes ${ }^{16}$, P.-O. Petruccis ${ }^{32}$, B. Peyaud ${ }^{18}$, Q. Piel ${ }^{24}$, S. Pita $^{31}$, V. Poireau ${ }^{24}$, D. A. Prokhorov ${ }^{23}$, H. Prokoph ${ }^{12}$, G. Pühlhofer ${ }^{29}$, M. Punch ${ }^{31,10}$, A. Quirrenbach ${ }^{25}$, S. Raab ${ }^{36}$, R. Rauth ${ }^{13}$, A. Reimer ${ }^{13}$, O. Reimer ${ }^{13}$, M. Renaud ${ }^{17}$, R. de los Reyes ${ }^{3}$, F. Rieger ${ }^{3,41}$, L. Rinchiuso ${ }^{18}$, C. Romoli ${ }^{4}$, G. Rowell ${ }^{14}$, B. Rudak ${ }^{34}$, C. B. Rulten ${ }^{15}$, V. Sahakian ${ }^{6,5}$, S. Saito ${ }^{42}$, D. A. Sanchez ${ }^{24}$, A. Santangelo ${ }^{29}$, M. Sasaki ${ }^{36}$, R. Schlickeiser ${ }^{11}$, F. Schüssler ${ }^{18}$, A. Schulz ${ }^{37}$, U. Schwanke ${ }^{7}$, S. Schwemmer ${ }^{25}$, M. Seglar-Arroyo ${ }^{18}$, A. S. Seyffert ${ }^{1}$, N. Shafi ${ }^{23}$, I. Shilon ${ }^{36}$, K. Shiningayamwe ${ }^{8}$, R. Simoni ${ }^{9}$, H. Sol ${ }^{15}$, F. Spanier ${ }^{1}$, M. Spir-Jacob ${ }^{31}$, Ł. Stawarz ${ }^{38}$, R. Steenkamp ${ }^{8}$, C. Stegmann ${ }^{35,37}$, C. Steppa ${ }^{35}$, I. Sushch ${ }^{1}$, T. Takahashi ${ }^{43}$, J.-P. Tavernet ${ }^{16}$, T. Tavernier ${ }^{18}$,

A. M. Taylor ${ }^{37}$, R. Terrier ${ }^{31}$, L. Tibaldo ${ }^{3}$, D. Tiziani ${ }^{36}$, M. Tluczykont ${ }^{2}$, C. Trichard ${ }^{20}$, M. Tsirou $^{17}$, N. Tsuji ${ }^{42}$, R. Tuffs ${ }^{3}$, Y. Uchiyama ${ }^{42}$, D. J. van der Walt ${ }^{1}$,

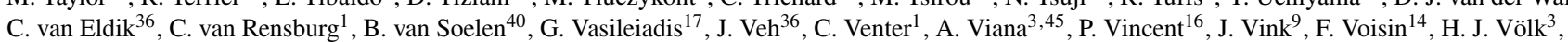
T. Vuillaume ${ }^{24}$, Z. Wadiasingh ${ }^{1}$, S. J. Wagner ${ }^{25}$, P. Wagner ${ }^{7}$, R. M. Wagner ${ }^{27}$, R. White ${ }^{3}$, A. Wierzcholska ${ }^{21}$, P. Willmann ${ }^{36}$, A. Wörnlein $^{36}$, D. Wouters ${ }^{18}$, R. Yang ${ }^{3}$, D. Zaborov ${ }^{30}$, M. Zacharias ${ }^{1}$, R. Zanin ${ }^{3}$, A. A. Zdziarski ${ }^{34}$, A. Zech ${ }^{15}$, F. Zefi $^{30}$, A. Ziegler ${ }^{36}$, J. Zorn ${ }^{3}$, and N. Żywucka ${ }^{38}$

(Affiliations can be found after the references)

Received 6 December 2017 / Accepted 4 January 2018

\begin{abstract}
Context. Recently, the high-energy (HE, $0.1-100 \mathrm{GeV}$ ) $\gamma$-ray emission from the object LMC P3 in the Large Magellanic Cloud (LMC) has been discovered to be modulated with a 10.3-day period, making it the first extra-galactic $\gamma$-ray binary.

Aims. This work aims at the detection of very-high-energy (VHE, $>100 \mathrm{GeV}) \gamma$-ray emission and the search for modulation of the VHE signal with the orbital period of the binary system.

Methods. LMC P3 has been observed with the High Energy Stereoscopic System (H.E.S.S.); the acceptance-corrected exposure time is $100 \mathrm{~h}$. The data set has been folded with the known orbital period of the system in order to test for variability of the emission. Results. VHE $\gamma$-ray emission is detected with a statistical significance of $6.4 \sigma$. The data clearly show variability which is phase-locked to the orbital period of the system. Periodicity cannot be deduced from the H.E.S.S. data set alone. The orbit-averaged luminosity in the $1-10 \mathrm{TeV}$ energy range is $(1.4 \pm 0.2) \times 10^{35} \mathrm{erg} \mathrm{s}^{-1}$. A luminosity of $(5 \pm 1) \times 10^{35} \mathrm{erg} \mathrm{s}^{-1}$ is reached during $20 \%$ of the orbit. HE and VHE $\gamma$-ray emissions are anti-correlated. LMC P3 is the most luminous $\gamma$-ray binary known so far.
\end{abstract}

Key words. gamma rays: stars - binaries: general - stars: massive

\section{Introduction}

More than $60 \%$ of all stellar systems containing high-mass stars (spectral type B2 or earlier) are binary or multiple systems (Duchêne \& Kraus 2013). When the more massive of the stars in these systems ends its life in a supernova explosion, a binary system is left behind where a compact object, either a neutron star or a black hole, is orbiting the remaining star. If these objects radiate most of their power at energies of more than $1 \mathrm{MeV}$ then they are called $\gamma$-ray binaries. The $\gamma$-ray emission arises either

${ }^{\star}$ Corresponding authors: H.E.S.S. Collaboration, e-mail: contact.hess@hess-experiment.eu from the interaction of a pulsar wind (driven by the rotational energy loss of a rotating neutron star) with the stellar wind, or from accretion of the stellar wind onto a black hole or neutron star. The companion stars in these systems are either $\mathrm{O}-$ or Betype stars. Only six $\gamma$-ray binaries have been identified so far. The nature of the compact objects is generally unknown, with the exception of PSR B1259-63/LS 2883 where the detection of pulsed emission (Johnston et al. 1992) shows that the compact object is a neutron star. A review of $\gamma$-ray binaries and their properties is given by Dubus $(2013,2015)$.

In order to identify previously undetected $\gamma$-ray binaries, Corbet et al. (2016) performed a search for periodic emission from the sources in the Fermi-LAT 3FGL catalogue 
(Acero et al. 2015). They found that the high-energy (HE, 0.1$100 \mathrm{GeV}$ ) $\gamma$-ray signal of LMC P3, an unidentified $\gamma$-ray source located in the Large Magellanic Cloud (LMC; Ackermann et al. 2016), is periodic with a period of $10.301 \pm 0.002$ days. In this publication, the phase zero of this system is defined to correspond to the maximum of the HE $\gamma$-ray emission at MJD $57410.25 \pm 0.34$. The position of LMC P3 is consistent with the one of the soft X-ray source CAL 60 (Long et al. 1981). Crampton et al. (1985) identified a star of spectral type O5 III(f) as the likely counterpart of this X-ray source. Subsequent X-ray observations with XMM-Newton (Bamba et al. 2006) and Chandra (Seward et al. 2012) confirmed a point-like X-ray source which is named CXOU 053600.0-673507. Seward et al. (2012) already concluded from the variabilities of the X-ray flux and the radial velocity of Balmer absorption lines that this object is likely a binary system. LMC P3 is located in the supernova remnant DEM L241, making it the third X-ray binary found in an observable supernova remnant after SS433/W 50 (Dubner et al. 1998) and SXP 1062 (Hénault-Brunet et al. 2012).

Little is known about the orbital parameters of the system. The most precise measurement of the orbital period comes from the HE $\gamma$-ray emission. Corbet et al. (2016) also analysed the radial velocity of the star and found an orbital period of 10.1 days and a superior conjunction of the companion star at MJD $57408.61 \pm 0.28$. The mass function prefers a neutron star as the compact object for a wide range of inclinations, but a black hole cannot be ruled out. The X-ray and radio emission of this object is modulated with the 10.3-day period, but is out of phase with the $\gamma$-ray emission (Corbet et al. 2016).

The detection of periodic $\gamma$-ray emission from a system with an O5-type companion star and the $\gamma$-ray to X-ray luminosity ratio allow a clear classification of this object as a high-mass $\gamma$-ray binary (Corbet et al. 2016). It is the first such object discovered outside the Milky Way. With an HE $\gamma$-ray luminosity in the energy range from $200 \mathrm{MeV}$ to $100 \mathrm{GeV}$ of $2.5 \times 10^{36} \mathrm{erg} \mathrm{s}^{-1}$ (Ackermann et al. 2016) it is also the most luminous $\gamma$-ray binary known so far.

\section{H.E.S.S. observations and results}

The LMC has been observed extensively with the High Energy Stereoscopic System (H.E.S.S.) since 2004. These observations led to the discovery of three individual very-high-energy (VHE, $>100 \mathrm{GeV}$ ) $\gamma$-ray-emitting sources (H.E.S.S. Collaboration et al. 2012, 2015a). H.E.S.S. is a system of five Imaging Air Cherenkov Telescopes, located in the Khomas Highland of Namibia at an altitude of $1800 \mathrm{~m}$. It is sensitive to $\gamma$ rays of energies from tens of $\mathrm{GeV}$ up to several tens of $\mathrm{TeV}$. The arrival direction of individual $\gamma$ rays can be reconstructed with an angular resolution of better than $0.1^{\circ}$, and their energy is estimated with a relative uncertainty of $15 \%$. The data discussed here were taken between 2004 and the beginning of 2016 and add up to a total observation time of $277 \mathrm{~h}$, almost $70 \mathrm{~h}$ more than what was used in the previous publication of LMC sources (H.E.S.S. Collaboration et al. 2015a). After correcting for dead time and camera offset angles, the effective (on-axis equivalent) exposure time for LMC P3 is $100 \mathrm{~h}$. About $5 \%$ of these observations were taken with the participation of the large H.E.S.S.-II telescope. The data recorded with this telescope are ignored in this analysis in order to obtain a homogeneous data set. The data were analysed using Model analysis with high-resolution cuts (de Naurois \& Rolland 2009), where the camera images are compared with a semi-analytical model using a log-likelihood minimisation technique. The results were cross-checked with

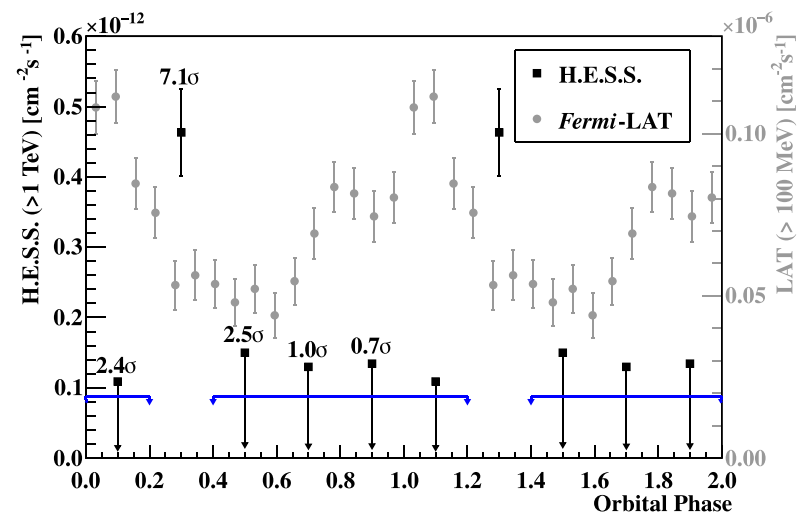

Fig. 1. Folded $\gamma$-ray light curves with orbital phase zero at the maximum of the HE $\gamma$-ray emission (MJD 57410.25 ). For better readability two orbits are shown. The black points (left $y$-axis) represent the VHE light curve (this work), the grey points (right $y$-axis) represent the $\mathrm{HE}$ light curve (from Corbet et al. 2016, Fig. 3). Error bars represent $1 \sigma$ statistical uncertainty. For the phase bins without significant detection, upper limits (at 95\% confidence level) are given. The labels at the data points indicate the statistical significance of the excess in each phase bin. The blue horizontal lines denote the upper limit (at 95\% confidence level) on the flux in the off-peak region (orbital phase 0.4 to 1.2).

an independent multi-variate analysis chain based on image parametrisation (Ohm et al. 2009; Lu 2013). The background was estimated from rings around the on regions to generate the $\gamma$-ray images (ring background, Berge et al. 2007) and from test regions with similar offsets from the camera centre for the spectral analysis (reflected background, Berge et al. 2007). Due to the large zenith and offset angles as well as the event selection cuts the energy threshold for this data set is $714 \mathrm{GeV}$.

At the nominal position of CXOU 053600.0-673507 an excess of $76.3 \gamma$-ray events is detected with a statistical significance of $6.4 \sigma$ (Table 1). The sensitivity of H.E.S.S. does not allow a detection of flux variations of the object on a nightly basis. Therefore, the nightly light curve of the emission does not show any sign of variability; the fit of a constant yields $\chi^{2}=75.3$ for 99 degrees of freedom. The search for periodic emission using a Lomb-Scargle test (Lomb 1976; Scargle 1982) and the Z-Transformed Discrete Correlation Function (Alexander 1997) does not show significant periodicity. Figure 1 shows the light curve folded with the orbital period of the system of 10.301 days, where orbital phase zero is defined as the maximum of the HE light curve at MJD 57410.25 (Corbet et al. 2016). Significant emission is detected only in the orbital phase bin between 0.2 and 0.4 with a pre-trial significance of $7.1 \sigma$. This corresponds to a post-trial significance of $6.9 \sigma$ after correcting for the test of five independent phase bins. All other phase bins do not show significant emission (significances less than $2.5 \sigma$, see Fig. 1). All phase bins have roughly the same exposure (between 18 and $21 \mathrm{~h}$ ). Fitting the folded light curve with a constant results in a $\chi^{2}$ value of 27.03 for 4 degrees of freedom. The $\chi^{2}$ probability that the folded light curve is constant is hence less than $1.95 \times 10^{-5}$. The emission is clearly variable and it is phase-locked to the orbital period of the system. Therefore, the detected VHE $\gamma$-ray emission can be associated to the binary system LMC P3.

Figure 2 shows the VHE $\gamma$-ray excess maps in the on-peak (orbital phase 0.2 to 0.4 ) and the off-peak (orbital phase 0.4 to 1.2) parts of the orbit. Fitting a point-like source folded with the instrument's point spread function results in a best-fit position of the source at $\mathrm{RA}=5^{\mathrm{h}} 36^{\mathrm{m}} 0^{\mathrm{s}}$, Dec $=-67^{\circ} 35^{\prime} 11^{\prime \prime}$, equinox J2000, with a statistical uncertainty of $\pm 23^{\prime \prime}$ in each direction; the source is hence labelled HESS J0536-675. The best-fit position 
H.E.S.S. Collaboration: Detection of variable VHE $\gamma$-ray emission from the extra-galactic $\gamma$-ray binary LMC P3
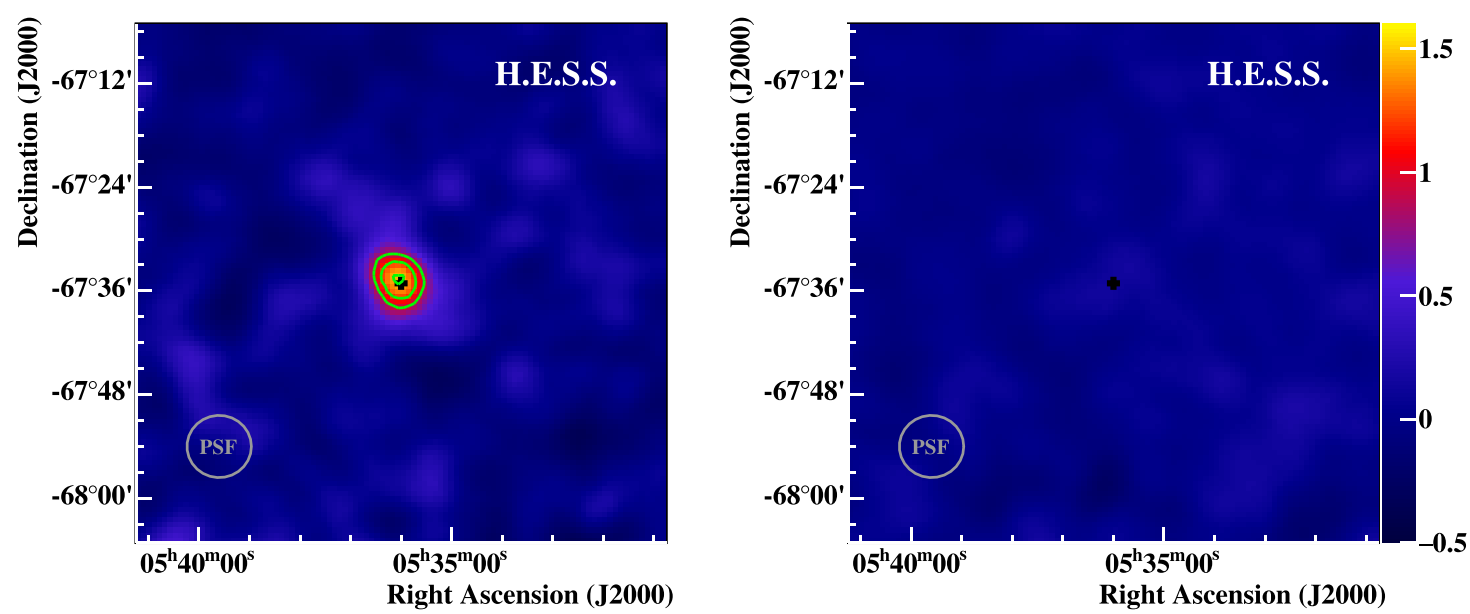

Fig. 2. H.E.S.S. excess count rate maps for the on-peak (left panel) and off-peak (right panel) regions of the orbit. The excess is smoothed with the point-spread function of the instrument $\left(68 \%\right.$ containment radius of $0.06^{\circ}$, indicated by the grey circle). The cross indicates the test position. Both plots have the same range, and the colour bar on the right-hand side is in units of excess counts per hour. The overlaid green contours represent 4 , 5 , and $6 \sigma$ statistical significance.

Table 1. Statistical results and spectral parameters for different orbital phase bins of HESS J0536-675.

\begin{tabular}{lcccccc}
\hline \hline Orbital phase bin & Excess & Significance & $\begin{array}{c}\Phi_{1 \mathrm{TeV}} \\
{\left[10^{-13} \mathrm{~cm}^{-2} \mathrm{~s}^{-1} \mathrm{TeV}^{-1}\right]}\end{array}$ & $\Gamma$ & $\begin{array}{c}F(>1 \mathrm{TeV}) \\
{\left[10^{-13} \mathrm{~cm}^{-2} \mathrm{~s}^{-1}\right]}\end{array}$ & $\begin{array}{c}L(1-10 \mathrm{TeV}, 50 \mathrm{kpc}) \\
{\left[10^{35} \mathrm{erg} \mathrm{s}^{-1}\right]}\end{array}$ \\
\hline Full orbit & 76.3 & $6.4 \sigma$ & $2.0 \pm 0.4$ & $2.5 \pm 0.2$ & $1.4 \pm 0.4$ & $1.4 \pm 0.2$ \\
On-peak & 41.1 & $7.1 \sigma$ & $5 \pm 1$ & $2.1 \pm 0.2$ & $5 \pm 2$ & $5 \pm 1$ \\
Off-peak & 35.0 & $3.3 \sigma$ & - & 2.4 (fixed) & $<0.88(95 \%$ CL) & $<0.88$ (95\% CL)
\end{tabular}

Notes. The on-peak region covers orbital phases from 0.2 to 0.4 , the off-peak region the orbital phases from 0.4 to 1.2. Orbital phase zero is at the maximum of the HE $\gamma$-ray emission (MJD 57 410.25).

is $4^{\prime \prime}$ (17\% of the statistical uncertainty) away from the nominal binary position. Therefore, the VHE $\gamma$-ray source is positionally compatible with the binary system.

Figure 3 shows the spectral energy distribution for the full orbit and the on-peak part of the orbit. The spectra are fitted with a simple power law, $\frac{\mathrm{d} N}{\mathrm{~d} E}=\Phi_{1 \mathrm{TeV}}\left(\frac{E}{1 \mathrm{TeV}}\right)^{-\Gamma}$, and the best-fit parameters are summarised in Table 1 . Significant emission is detected up to at least $10 \mathrm{TeV}$ and no high-energy cut-off of the spectra has been detected. For the off-peak region of the orbit an upper limit on the integrated $\gamma$-ray flux has been obtained. Most of the $\gamma$-ray emission is radiated during only $20 \%$ of the binary's orbit, when it reaches a flux of about four times the orbit-averaged flux.

\section{Discussion}

The emission from HESS J0536-675 is variable and modulated with the orbital period of the binary system, indicating that the size of the $\gamma$-ray emission region is at most the size of the binary system. The companion star in this system is of the type O5III, with a typical mass of $40 M_{\odot}$ (Martins et al. 2005). For a neutron star with a mass of $1.4 M_{\odot}$ as the compact object the semi-major axis of the orbit would be $0.32 \mathrm{AU}$.

The two scenarios proposed for $\gamma$-ray binaries are that the $\gamma$-ray emission can be powered either by the spin-down of a pulsar or by accretion of the stellar wind onto the compact object. In the pulsar wind scenario, electrons are accelerated in the vicinity of the pulsar or in the shock front between the pulsar wind and stellar wind. The relativistic electrons produce $\gamma$-ray emission by inverse Compton (IC) upscattering of stellar photons. A typical O5III star has a surface temperature of $40 \mathrm{kK}$ and a luminosity of $5.4 \times 10^{5} L_{\odot}$ (Martins et al. 2005). The IC scattering is in the Klein-Nishina regime and a hard electron spectrum with an index of -1.5 is required to produce the observed $\gamma$-ray spectrum. For a binary separation of 0.32 AU the stellar photon field has an energy density of $253 \mathrm{erg} \mathrm{cm}^{-3}$. Electrons with energies between 0.5 and $50 \mathrm{TeV}$ and a total energy of $2.5 \times 10^{38} \mathrm{erg}$ are required to produce the observed $\gamma$-ray emission. The IC cooling time (Khangulyan et al. 2008, Eq. (1)) of TeV electrons in this photon field is of the order of $100 \mathrm{~s}$. Therefore, the pulsar's spindown power needs to be at least $10^{36} \mathrm{erg} \mathrm{s}^{-1}$ in order to provide the energy for the observed VHE $\gamma$-ray emission alone.

In the accretion scenario, a situation that is encountered in microquasars, the stellar wind of the companion star is accreted onto the compact object and gravitational potential energy is released as radiation. The accretion luminosity of a neutron star is

$$
L_{\mathrm{acc}}=\left(\frac{\dot{M}}{10^{-10} M_{\odot} \mathrm{yr}^{-1}}\right)\left(\frac{M_{\mathrm{co}}}{1.4 M_{\odot}}\right)\left(\frac{10 \mathrm{~km}}{R_{\mathrm{co}}}\right) \times 1.2 \times 10^{36} \mathrm{erg} \mathrm{s}^{-1},
$$

where $M_{\text {co }}$ and $R_{\text {co }}$ are the mass and radius of the compact object (Frank et al. 1992). $\dot{M}$ is the mass-accretion rate which is of the order of $10^{-10} M_{\odot} \mathrm{yr}^{-1}$ in a typical close binary system with accretion of the stellar wind. Accretion can power the observed $\gamma$-ray emission provided that the conversion efficiency from accretion power to $\gamma$ rays approaches unity. Much higher mass-accretion rates and thus a higher accretion luminosity can be achieved by Roche lobe overflow, for instance an accretion rate of $10^{-4} M_{\odot} \mathrm{yr}^{-1}$ is discussed for SS 443 in such a scenario (for a review see Fabrika 2004). A more massive compact object can also increase the accretion luminosity by a factor of a few.

The peak of the VHE emission at phases between 0.2 and 0.4 coincides with the decline of the HE $\gamma$-ray emission towards its broad minimum between orbital phases 0.3 and 0.7 (see Fig. 1). 


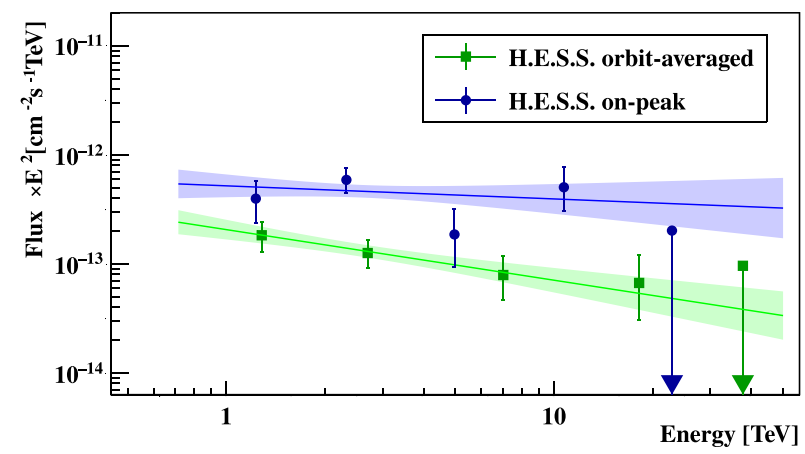

Fig. 3. Spectral energy distribution averaged over the full orbit (green, squares) and for the on-peak orbital phase range (orbital phase from 0.2 to 0.4 : blue, circles). The data points have $1 \sigma$ statistical error bars, upper limits are for a $95 \%$ confidence level. The best fit and its uncertainty are represented by the solid lines and shaded areas, respectively.

The variability of the $\gamma$-ray flux over the orbit of the system can be explained by the angle-dependent cross section of IC scattering, $\gamma \gamma$ absorption or the eccentricity of the orbit. IC scattering is most efficient in head-on collisions in which the low-energy photons are backscattered. Therefore, the maximum of the $\gamma$-ray emission is expected at superior conjunction. Similarly, the minimum of the $\gamma$-ray emission occurs at inferior conjunction, when only tail-on collisions are observed. Further on, $\gamma \gamma$ absorption due to pair-production (Gould \& Schréder 1967) can modulate the VHE $\gamma$-ray emission with the orbital period and the flux maximum is expected at inferior conjunction (Böttcher \& Dermer 2005). This scenario is discussed for LS 5039 (Abdo et al. 2009) and can naturally explain why the HE and VHE emissions of LMC P3 are out of phase. In such a scenario the superior conjunction of LMC P3 would be around orbital phase 0.0 and inferior conjunction would be between 0.2 and 0.4 . The varying separation in an eccentric orbit could also explain the variability of the $\gamma$-ray emission. Near periastron, the compact object probes a higher stellar photon-field density and a higher stellar-wind density. In order to vary the $\gamma$-ray flux by a factor of around 4 (the ratio between the off-peak upper limit and the on-peak flux) by either IC emission or stellar-wind accretion, the binary separation must vary by a factor of 2 , which is the case for an eccentricity of 0.33 . In this case, and considering only VHE $\gamma$-ray emission, periastron would be between orbital phases 0.2 and 0.4 .

HE and VHE $\gamma$-ray emission may also arise from two different particle populations. Zabalza et al. (2013) propose the apex of the contact discontinuity between the compact object and the star as the source of the HE emission, and the pulsar wind termination shock on the opposite side as the source of the VHE emission. These emission sites together with geometric effects of the binary's orbit can also explain the phase-shift of the HE and VHE $\gamma$-ray light curves.

LMC P3 is the sixth, and the most luminous, $\gamma$-ray binary discovered so far. With an O-type companion star it is similar to LS 5039 and 1FGL J1018.6-5856. With the HE and VHE emissions out of phase, it resembles LS 5039 (H.E.S.S. Collaboration et al. 2006; Abdo et al. 2009), which is in contrast to 1FGL J1018.6-5856 where HE and VHE emissions are in phase (Ackermann et al. 2012; H.E.S.S. Collaboration et al. 2015b). But contrary to LS 5039, which shows VHE $\gamma$-ray emission during more than $50 \%$ of the orbit, the duration of the emission of HESS J0536-675 covers less than 20\% of the orbit (given the current instrument sensitivity). The VHE light curve of LMC P3 is similar to the VHE $\gamma$-ray light curve of 1FGL J1018.6-5856 (H.E.S.S. Collaboration et al. 2015b). The detection of periodic
HE and VHE emission from LMC P3 helps to fill the zoo of $\gamma$-ray binaries and will help to understand the underlying particle acceleration and $\gamma$-ray production mechanisms. Detailed modelling of the HE and VHE light curves requires knowledge of the orbital parameters of the system.

The sensitivity of H.E.S.S. only allows the detection of VHE $\gamma$-ray radiation during the high-state of the emission. The $3.3 \sigma$ statistical significance during the off-peak part of the orbit may indicate that the VHE emission extends into this part of the orbit as well. The ten-fold sensitivity of the future Cherenkov Telescope Array (CTA; Hinton et al. 2013) will allow for the investigation of this part of the orbit of the binary system.

\section{Conclusions}

Variable VHE $\gamma$-ray emission from the newly discovered binary system LMC P3 has been detected with H.E.S.S. The emission is phase-locked to the orbital period of the system. This makes HESS J0536-675 the sixth VHE $\gamma$-ray-emitting binary and the first extra-galactic $\gamma$-ray binary. The energy spectrum of the VHE $\gamma$-ray emission is described by a simple power law. The orbit-averaged VHE luminosity of the system is $(1.4 \pm 0.2) \times 10^{35} \mathrm{erg} \mathrm{s}^{-1}$. During $20 \%$ of the orbit the VHE luminosity reaches $(5 \pm 1) \times 10^{35} \mathrm{erg} \mathrm{s}^{-1}$. This makes HESS J0536-675 the most luminous $\gamma$-ray binary known to date. The VHE $\gamma$-ray emission can be either powered by the spin-down of a pulsar or by accretion of the stellar wind onto the compact object. A luminous pulsar $\left(\dot{E} \geq 10^{36} \mathrm{erg} \mathrm{s}^{-1}\right)$, a high mass-accretion rate or a very massive compact object are needed to provide the energy for the observed VHE $\gamma$-ray emission. The VHE emission is out of phase with the HE emission which may be explained by absorption due to pair production, or by different particle distributions responsible for the HE and VHE $\gamma$-ray production. Observations with CTA may lead to detection of $\gamma$-ray emission during the off-peak part of the orbit allowing the modelling of the entire light curve.

Acknowledgements. The support of the Namibian authorities and of the University of Namibia in facilitating the construction and operation of H.E.S.S. is gratefully acknowledged, as is the support by the German Ministry for Education and Research (BMBF), the Max Planck Society, the German Research Foundation (DFG), the Alexander von Humboldt Foundation, the Deutsche Forschungsgemeinschaft, the French Ministry for Research, the CNRS-IN2P3 and the Astroparticle Interdisciplinary Programme of the CNRS, the U.K. Science and Technology Facilities Council (STFC), the IPNP of the Charles University, the Czech Science Foundation, the Polish National Science Centre, the South African Department of Science and Technology and National Research Foundation, the University of Namibia, the National Commission on Research, Science \& Technology of Namibia (NCRST), the Innsbruck University, the Austrian Science Fund (FWF), and the Austrian Federal Ministry for Science, Research and Economy, the University of Adelaide and the Australian Research Council, the Japan Society for the Promotion of Science and by the University of Amsterdam. We appreciate the excellent work of the technical support staff in Berlin, Durham, Hamburg, Heidelberg, Palaiseau, Paris, Saclay, and in Namibia in the construction and operation of the equipment. This work benefited from services provided by the H.E.S.S. Virtual Organisation, supported by the national resource providers of the EGI Federation. We thank Robin Corbet for providing the HE light curve data shown in Fig. 1.

\section{References}

Abdo, A. A., et al. (Fermi LAT Collaboration) 2009, ApJ, 706, L56 Acero, F., et al. (Fermi LAT Collaboration) 2015, ApJS, 218, 23 Ackermann, M., et al. (Fermi LAT Collaboration) 2012, Science, 335, 189 Ackermann, M., et al. (Fermi LAT Collaboration) 2016, A\&A, 586, A71 Alexander, T. 1997, in Astronomical Time Series, eds. D. Maoz, A. Sternberg, \& E. M. Leibowitz, Astrophys. Space Sci. Lib., 218, 163

Bamba, A., Ueno, M., Nakajima, H., Mori, K., \& Koyama, K. 2006, A\&A, 450, 585 
Berge, D., Funk, S., \& Hinton, J. 2007, A\&A, 466, 1219

Böttcher, M., \& Dermer, C. D. 2005, ApJ, 634, L81

Corbet, R. H. D., Chomiuk, L., Coe, M. J., et al. 2016, ApJ, 829, 105

Crampton, D., Cowley, A. P., Thompson, I. B., \& Hutchings, J. B. 1985, AJ, 90, 43

de Naurois, M., \& Rolland, L. 2009, Astropart. Phys., 32, 231

Dubner, G. M., Holdaway, M., Goss, W. M., \& Mirabel, I. F. 1998, AJ, 116, 1842

Dubus, G. 2013, A\&ARv, 21, 64

Dubus, G. 2015, C. R. Phys., 16, 661

Duchêne, G., \& Kraus, A. 2013, ARA\&A, 51, 269

Fabrika, S. 2004, Astrophys. Space Phys. Rev., 12, 1

Frank, J., King, A., \& Raine, D. 1992, Accretion Power in Astrophysics (Cambridge, UK: Cambridge University Press)

Gould, R. J., \& Schréder, G. P. 1967, Phys. Rev., 155, 1404

Hénault-Brunet, V., Oskinova, L. M., Guerrero, M. A., et al. 2012, MNRAS, 420 , L13

H.E.S.S. Collaboration, Aharonian, F., Akhperjanian, A. G., et al. 2006, A\&A, 460,743

H.E.S.S. Collaboration, Abramowski, A., Acero, F., et al. 2012, A\&A, 545, L2

H.E.S.S. Collaboration, Abramowski, A., Aharonian, F., et al. 2015a, Science, 347,406

H.E.S.S. Collaboration, Abramowski, A., Aharonian, F., et al. 2015b, A\&A 577, A131

Hinton, J., Sarkar, S., Torres, D., \& Knapp, J. 2013, Astropart. Phys., 43, 1

Johnston, S., Manchester, R. N., Lyne, A. G., et al. 1992, ApJ, 387, L37

Khangulyan, D., Aharonian, F., \& Bosch-Ramon, V. 2008, MNRAS, 383, 467

Lomb, N. R. 1976, Ap\&SS, 39, 447

Long, K. S., Helfand, D. J., \& Grabelsky, D. A. 1981, ApJ, 248, 925

Lu, C. C. 2013, in Proc. 33rd Int. Cosmic Ray Conf. (ICRC2013), Rio de Janeiro, Brazil, July 2-9, 2013, 1105

Martins, F., Schaerer, D., \& Hillier, D. J. 2005, A\&A, 436, 1049

Ohm, S., van Eldik, C., \& Egberts, K. 2009, Astropart. Phys., 31, 383

Scargle, J. D. 1982, ApJ, 263, 835

Seward, F. D., Charles, P. A., Foster, D. L., et al. 2012, ApJ, 759, 123

Zabalza, V., Bosch-Ramon, V., Aharonian, F., \& Khangulyan, D. 2013, A\&A, 551, A 17

1 Centre for Space Research, North-West University, Potchefstroom 2520, South Africa

2 Universität Hamburg, Institut für Experimentalphysik, Luruper Chaussee 149, 22761 Hamburg, Germany

3 Max-Planck-Institut für Kernphysik, PO Box 103980, 69029 Heidelberg, Germany

4 Dublin Institute for Advanced Studies, 31 Fitzwilliam Place, Dublin 2, Ireland

5 National Academy of Sciences of the Republic of Armenia, Marshall Baghramian Avenue, 24, 0019 Yerevan, Armenia

6 Yerevan Physics Institute, 2 Alikhanian Brothers St., 375036 Yerevan, Armenia

7 Institut für Physik, Humboldt-Universität zu Berlin, Newtonstr. 15, 12489 Berlin, Germany

8 University of Namibia, Department of Physics, Private Bag 13301, Windhoek, Namibia

9 GRAPPA, Anton Pannekoek Institute for Astronomy, University of Amsterdam, Science Park 904, 1098 XH Amsterdam, The Netherlands

10 Department of Physics and Electrical Engineering, Linnaeus University, 35195 Växjö, Sweden

11 Institut für Theoretische Physik, Lehrstuhl IV: Weltraum und Astrophysik, Ruhr-Universität Bochum, 44780 Bochum, Germany

12 GRAPPA, Anton Pannekoek Institute for Astronomy and Institute of High-Energy Physics, University of Amsterdam, Science Park 904, 1098 XH Amsterdam, The Netherlands

13 Institut für Astro- und Teilchenphysik, Leopold-FranzensUniversität Innsbruck, 6020 Innsbruck, Austria

14 School of Physical Sciences, University of Adelaide, Adelaide 5005, Australia

15 LUTH, Observatoire de Paris, PSL Research University, CNRS, Université Paris Diderot, 5 Place Jules Janssen, 92190 Meudon, France
16 Sorbonne Universités, UPMC Université Paris 06, Université Paris Diderot, Sorbonne Paris Cité, CNRS, Laboratoire de Physique Nucléaire et de Hautes Energies (LPNHE), 4 place Jussieu, 75252 Paris Cedex 5, France

17 Laboratoire Univers et Particules de Montpellier, Université Montpellier, CNRS/IN2P3, CC 72, Place Eugène Bataillon, 34095 Montpellier Cedex 5, France

18 IRFU, CEA, Université Paris-Saclay, 91191 Gif-sur-Yvette, France

19 Astronomical Observatory, The University of Warsaw, Al. Ujazdowskie 4, 00-478 Warsaw, Poland

20 Aix Marseille Université, CNRS/IN2P3, CPPM, Marseille, France

21 Instytut Fizyki Ja̧drowej PAN, ul. Radzikowskiego 152, 31-342 Kraków, Poland

22 Funded by EU FP7 Marie Curie, Grant Agreement No. PIEF-GA2012-332350

23 School of Physics, University of the Witwatersrand, 1 Jan Smuts Avenue, Braamfontein, Johannesburg 2050, South Africa

24 Laboratoire d'Annecy de Physique des Particules, Université Savoie Mont-Blanc, CNRS/IN2P3, 74941 Annecy-le-Vieux, France

25 Landessternwarte, Universität Heidelberg, Königstuhl, 69117 Heidelberg, Germany

26 Université Bordeaux, CNRS/IN2P3, Centre d'Études Nucléaires de Bordeaux Gradignan, 33175 Gradignan, France

27 Oskar Klein Centre, Department of Physics, Stockholm University, Albanova University Center, 10691 Stockholm, Sweden

28 Wallenberg Academy Fellow

29 Institut für Astronomie und Astrophysik, Universität Tübingen, Sand 1, 72076 Tübingen, Germany

30 Laboratoire Leprince-Ringuet, Ecole Polytechnique, CNRS/IN2P3, 91128 Palaiseau, France

31 APC, AstroParticule et Cosmologie, Université Paris Diderot, CNRS/IN2P3, CEA/Irfu, Observatoire de Paris, Sorbonne Paris Cité, 10, rue Alice Domon et Léonie Duquet, 75205 Paris Cedex 13, France

32 Univ. Grenoble Alpes, CNRS, IPAG, 38000 Grenoble, France

33 Department of Physics and Astronomy, The University of Leicester, University Road, Leicester LE1 7RH, UK

34 Nicolaus Copernicus Astronomical Center, Polish Academy of Sciences, ul. Bartycka 18, 00-716 Warsaw, Poland

35 Institut für Physik und Astronomie, Universität Potsdam, KarlLiebknecht-Strasse 24/25, 14476 Potsdam, Germany

36 Friedrich-Alexander-Universität Erlangen-Nürnberg, Erlangen Centre for Astroparticle Physics, Erwin-Rommel-Str. 1, 91058 Erlangen, Germany

37 DESY, 15738 Zeuthen, Germany

38 Obserwatorium Astronomiczne, Uniwersytet Jagielloński, ul. Orla 171, 30-244 Kraków, Poland

39 Centre for Astronomy, Faculty of Physics, Astronomy and Informatics, Nicolaus Copernicus University, Grudziadzka 5, 87-100 Torun, Poland

40 Department of Physics, University of the Free State, PO Box 339, Bloemfontein 9300, South Africa

41 Heisenberg Fellow (DFG), ITA Universität Heidelberg, Germany

42 Department of Physics, Rikkyo University, 3-34-1 Nishi-Ikebukuro, Toshima-ku, Tokyo 171-8501, Japan

43 Japan Aerospace Exploration Agency (JAXA), Institute of Space and Astronautical Science (ISAS), 3-1-1 Yoshinodai, Chuo-ku, Sagamihara, Kanagawa 229-8510, Japan

44 Now at the School of Physics, The University of New South Wales, Sydney 2052, Australia

45 Now at Instituto de Física de São Carlos, Universidade de São Paulo, Av. Trabalhador São-carlense, 400 - CEP 13566-590, São Carlos, SP, Brazil

46 Now at Kavli Institute for Particle Astrophysics and Cosmology, Department of Physics and SLAC National Accelerator Laboratory, Stanford University, Stanford, CA 94305, USA 